\title{
Measurement of the High Energy Two-Body Deuteron Photodisintegration Differential Cross Section
}

E. C. Schulte, ${ }^{1}$ A. Ahmidouch, ${ }^{2}$ C. S. Armstrong, ${ }^{3}$ J. Arrington, ${ }^{4}$ R. Asaturyan, ${ }^{5}$ S. Avery, ${ }^{6}$ O. K. Baker, ${ }^{3,6}$ D. H. Beck, ${ }^{1}$ H. P. Blok, ${ }^{7}$ C. W. Bochna, ${ }^{1}$ W. Boeglin, ${ }^{8}$ P. Y. Bosted, ${ }^{9}$ M. Bouwhuis, ${ }^{1}$ H. Breuer, ${ }^{10}$ D. S. Brown, ${ }^{10}$ A. Bruell, ${ }^{11}$ R. V. Cadman, ${ }^{1}$ R. Carlini, ${ }^{3}$ N. S. Chant, ${ }^{10}$ A. Cochran, ${ }^{6}$ L. Cole,${ }^{6}$ S. Danagoulian, ${ }^{2,3}$ D. B. Day, ${ }^{12}$ J. A. Dunne, ${ }^{13}$ D. Dutta, ${ }^{11}$ R. Ent, ${ }^{3}$ H. C. Fenker, ${ }^{3}$ B. Fox,${ }^{14}$ L. Gan, ${ }^{6}$ H. Gao, ${ }^{11}$ K. Garrow, ${ }^{3}$ D. Gaskell, ${ }^{4}$ A. Gasparian, ${ }^{6}$ D. F. Geesaman, ${ }^{4}$ R. Gilman, ${ }^{3,15}$ C. Glashausser, ${ }^{15}$ P. Gueye, ${ }^{6}$ M. Harvey, ${ }^{6}$ R. J. Holt ${ }^{1,} *$ H. E. Jackson, ${ }^{4}$ X. Jiang, ${ }^{15}$ C.E. Keppel, ${ }^{3,6}$ E. R. Kinney, ${ }^{14}$ Y. Liang, ${ }^{9}$ W. Lorenzon, ${ }^{17}$ A. F. Lung, ${ }^{3}$ D. J. Mack, ${ }^{3}$ P. E. Markowitz, ${ }^{8}$ J. Martin, ${ }^{11}$ K. McIlhany, ${ }^{11}$ D. McKee, ${ }^{18}$ D. G. Meekins, ${ }^{19}$ M. A. Miller, ${ }^{1}$ R. G. Milner,${ }^{11}$ J. H. Mitchell, ${ }^{3}$ H. Mkrtchyan, ${ }^{5}$ B. A. Mueller, ${ }^{4}$ A. M. Nathan, ${ }^{1}$ G. Niculescu, ${ }^{20}$ I. Niculescu, ${ }^{21}$ T. G. O’Neill,,${ }^{4}$ V. Papavassiliou, ${ }^{18}$ S. F. Pate, ${ }^{18}$ R. B. Piercey, ${ }^{13}$ D. H. Potterveld, ${ }^{4}$ R. D. Ransome, ${ }^{15}$ J. Reinhold, ${ }^{8}$ E. Rollinde,${ }^{3}$ P. Roos, ${ }^{10}$ A. Saha, ${ }^{3}$ A. J. Sarty, ${ }^{19, \dagger}$ R. Sawafta, ${ }^{2}$ E. Segbefia,${ }^{6}$ T. Shin, ${ }^{11}$ S. Stepanyan, ${ }^{5}$ S. Strauch,${ }^{15}$ M. F. Sutter, ${ }^{11}$ V. Tadevosyan, ${ }^{5}$ L. Tang, ${ }^{6}$ R. Tieulent, ${ }^{3}$ A. Uzzle, ${ }^{6}$ W. F. Vulcan, ${ }^{3}$ S. A. Wood,${ }^{3}$ F. Xiong, ${ }^{9}$ L. Yuan, ${ }^{6}$ M. Zeier ${ }^{12}$ B. Zihlmann, ${ }^{12}$ and V. Ziskin ${ }^{11}$

${ }^{1}$ University of Illinois at Urbana-Champaign, Urbana, Illinois 61801

${ }^{2}$ North Carolina A\&T State University, Greensboro, North Carolina 27411

${ }^{3}$ Thomas Jefferson National Accelerator Facility, Newport News, Virginia 23606

${ }^{4}$ Argonne National Laboratory, Argonne, Illinois 60439

${ }^{5}$ Yerevan Physics Institute, Yerevan, Armenia

${ }^{6}$ Hampton University, Hampton, Virginia 23668

${ }^{7}$ Vrije Universiteit, Amsterdam, The Netherlands

${ }^{8}$ Florida International University, University Park, Florida 33199

${ }^{9}$ University of Massachusetts, Amherst, Massachusetts 01003

${ }^{10}$ University of Maryland, College Park, Maryland 20742

${ }^{11}$ Massachusetts Institute of Technology, Cambridge, Massachusetts 02139

${ }^{12}$ University of Virginia, Charlottesville, Virginia 22901

${ }^{13}$ Mississippi State University, Starkville, Mississippi 39759

${ }^{14}$ University of Colorado, Boulder, Colorado 80309

${ }^{15}$ Rutgers University, New Brunswick, New Jersey 08903

${ }^{16}$ American University, Washington, D.C. 20016

${ }^{17}$ University of Michigan, Ann Arbor, Michigan 48109

${ }^{18}$ New Mexico State University, Las Cruces, New Mexico 88003

${ }^{19}$ Florida State University, Tallahassee, Florida 32306

${ }^{20}$ Ohio University, Athens, Ohio 45701

${ }^{21}$ George Washington University, Washington, D.C. 20052

(Received 4 June 2001; published 16 August 2001)

\begin{abstract}
The first measurements of the $d(\gamma, p) n$ differential cross section at forward angles and photon energies above $4 \mathrm{GeV}$ were performed at the Thomas Jefferson National Accelerator Facility (JLab). The results indicate evidence of an angular dependent scaling threshold. Results at $\theta_{\mathrm{cm}}=37^{\circ}$ are consistent with the constituent counting rules for $E_{\gamma} \gtrsim 4 \mathrm{GeV}$, while those at $70^{\circ}$ are consistent with the constituent counting rules for $E_{\gamma} \gtrsim 1.5 \mathrm{GeV}$.
\end{abstract}

DOI: $10.1103 /$ PhysRevLett.87.102302

PACS numbers: 13.75.Cs, 24.85.+p, 25.10. $+\mathrm{s}, 25.20 .-\mathrm{x}$

Understanding nuclear interactions in the few $\mathrm{GeV}$ energy region is among the major challenges facing nuclear physics. Existing meson-baryon descriptions of deuteron photodisintegration break down above a photon energy of approximately $1 \mathrm{GeV}$. This suggests that quarks and gluons, the degrees of freedom in quantum chromodynamics (QCD), may be a more appropriate basis for describing nuclear interactions in the few $\mathrm{GeV}$ energy region.

The $d(\gamma, p) n$ reaction is ideal for investigating the transition region from meson exchange to QCD because of its simplicity and amenability to calculation as well as high momentum transfer per nucleon [1]. SLAC data $[2,3]$ above $E_{\gamma}=1 \mathrm{GeV}$, later confirmed and extended by Jefferson Lab [4], exhibit scaling consistent with a quark gluon picture of deuteron photodisintegration for $E_{\gamma} \gtrsim 1 \mathrm{GeV}$ at $\theta_{\mathrm{cm}}=90^{\circ}$. Recent polarized proton results from deuteron photodisintegration at $\theta_{\mathrm{cm}}=90^{\circ}$ show an induced polarization inconsistent with meson exchange current (MEC) model predictions [5]. At forward angles, $37^{\circ}$ and $53^{\circ}$, however, scaling is not observed for $E_{\gamma} \lesssim 4.0 \mathrm{GeV}$. The present work provides new data for the $d(\gamma, p) n$ differential cross section up to $5.5 \mathrm{GeV}$ at 
forward angles in order to investigate the potential onset of scaling and provide benchmark data for future theoretical studies.

By including nucleon excited states, traditional models, which describe nucleon-nucleon interactions with meson exchange currents, have been extended into the few $\mathrm{GeV}$ energy region. At very high energies asymptotic behavior is expected and nucleon-nucleon interactions should be well described by the scaling behavior of perturbative QCD (pQCD) [6-8]. In the intermediate energy regime, where meson exchange models fail [5] and pQCD is not yet expected to be applicable, nonperturbative QCD methods, such as the quark exchange model, hard rescattering model (HRM) [9], and quark gluon string (QGS) model [10] should be considered.

The constituent counting rules, believed to apply for energies much greater than the mass of the particles in the process [7], predict a center-of-mass energy dependence for the $d(\gamma, p) n$ reaction given by

$$
\frac{d \sigma}{d t}(\gamma d \rightarrow p n) \sim \frac{1}{s^{11}} f\left(\theta_{\mathrm{cm}}\right) .
$$

In Eq. (1) $s$ and $t$ are the Mandelstam variables for the total center-of-mass energy squared $(s)$ and the momentum transfer squared $(t)$ in the $s$ channel. The function $f\left(\theta_{\mathrm{cm}}\right)$ gives the angular dependence of the differential cross section and depends upon the underlying reaction mechanism [7].

The reduced nuclear amplitude (RNA) [11] and quark exchange [12] models are similar to that of Brodsky and Chertok [6]. In these models the deuteron is viewed as a composite object containing two nucleons, each of which carries, on the average, one-half of the momentum of the nucleus. Internal structure of the nucleons is removed by dividing out the nucleon form factors from the scattering amplitude [11], which is described by the interchange of two quarks and the exchange of a hard gluon between the nucleons in the deuteron. This method gives excellent agreement with $e$ - $d$ elastic scattering [6], but not with the deuteron photodisintegration data for $E_{\gamma} \leq 4 \mathrm{GeV}$ [4].

The QGS model is based on Regge phenomenology. This model for deuteron photodisintegration uses two types of nonlinear Regge trajectories. The first has a square root nonlinear form and the second a logarithmic nonlinear form. Below $E_{\gamma}=4 \mathrm{GeV}$ the logarithmic form gives good agreement with energy and angular dependence [10].

In the HRM the long range behavior of the deuteron is described by a deuteron wave function. The photon couples to a quark in one of the nucleons. This coupling causes a high-momentum transfer interchange of the quark with a quark in the other nucleon. This exchange is described by high energy, large angle, neutron-proton scattering data [9]. The HRM gives a fair description of the energy dependence at $\theta_{\mathrm{cm}}=37^{\circ}$ and $53^{\circ}$ below $E_{\gamma}=4 \mathrm{GeV}$.

Traditional meson exchange models reproduce the data well for $E_{\gamma} \lesssim 1 \mathrm{GeV}$, but fail to do so at higher energies
$[13,14]$. In an effort to resolve this problem, the asymptotic meson exchange current (AMEC) model [15] deviates from traditional meson exchange by describing the nucleon-nucleon interaction in the deuteron with a form factor. At $\theta_{\mathrm{cm}}=37^{\circ}, 53^{\circ}$, and $90^{\circ}$ for $E_{\gamma} \leq 4 \mathrm{GeV}$, the energy dependence is in reasonable agreement with the data [4], but the cross sections must be independently normalized.

Jefferson Lab experiment E96-003 was performed in Hall $\mathrm{C}$ at the Continuous Electron Beam Accelerator Facility. A $2 \%$ or $4 \%$ radiation length $\mathrm{Cu}$ target was irradiated with 5.0 and $5.5 \mathrm{GeV}$ electrons to produce a beam of real bremsstrahlung photons. These photons impinged upon a $12.5 \mathrm{~cm}$ long cryogenic deuterium or hydrogen target [16]. The photoprotons were detected in the Hall C High Momentum Spectrometer (HMS). The HMS was chosen because of its high central momentum $\left(p_{0}=7.4 \mathrm{GeV} / c\right)$, large momentum acceptance $\left(\frac{\Delta p}{p_{0}}=\right.$ $\pm 8 \%)$, reasonable momentum resolution $(\approx 0.13 \%)$, and large solid angle $(\Delta \Omega \sim 6 \mathrm{msr})$ [17].

Measurements were taken at each of three center-ofmass angles, $\theta_{\mathrm{cm}}=37^{\circ}, 53^{\circ}$, and $70^{\circ}$, with the radiator always in the electron beam [18]. The recoil protons were tracked using drift chambers. Time-of-flight information was obtained by a pair of $x-y$ hodoscopes, separated by approximately $2.2 \mathrm{~m}$, which also served to form the trigger. The $1.2 \mathrm{~m}$ long Čerenkov counter filled with $0.78 \mathrm{~atm}$ of $C_{4} F_{10}$ gas was used to eliminate pions. In this experiment all central momenta were above $2.5 \mathrm{GeV} / c$, the threshold for pion detection with $C_{4} F_{10}$ at this pressure.

From the recoil proton momentum and angle, the photon energy spectrum, $E_{\gamma}$, was reconstructed. Only the highest energy protons, those with energies close to the bremsstrahlung end point, were used to calculate the $d(\gamma, p) n$ cross section. Data taken on the liquid hydrogen target were used to measure the background. Yield from the kinematically forbidden region above the end point in the hydrogen spectrum was scaled to match yield taken from the same region in the deuterium spectrum. The background represented an average of $30 \%$ of the yield. Systematic error due to the background subtraction method was estimated to be $\$ 3 \%$. The scaled hydrogen yield was subtracted from the deuterium to produce the photoproton yield (Fig. 1). This was then used to calculate the laboratory $d(\gamma, p) n$ differential cross section.

Figure 1 shows typical photon energy spectra after background subtraction for $\theta_{\mathrm{cm}}=37^{\circ}$ and $53^{\circ}$ at $5.5 \mathrm{GeV}$. These spectra have the expected shape, with the bremsstrahlung end point located at the beam energy, illustrated by the solid curve. The solid curve represents two calculated bremsstrahlung spectra, each convolved with a Gaussian energy resolution function, scaled by $s^{-10}$, and normalized to the data. The end point of the second spectrum is set to the kinematically determined threshold of the $\gamma+d \rightarrow p+n+\pi^{0}$ channel. The dashed curve in Fig. 1 is the extension of the two-body component of the solid curve. Yield from pion production at $\theta_{\mathrm{cm}}=37^{\circ}$ 


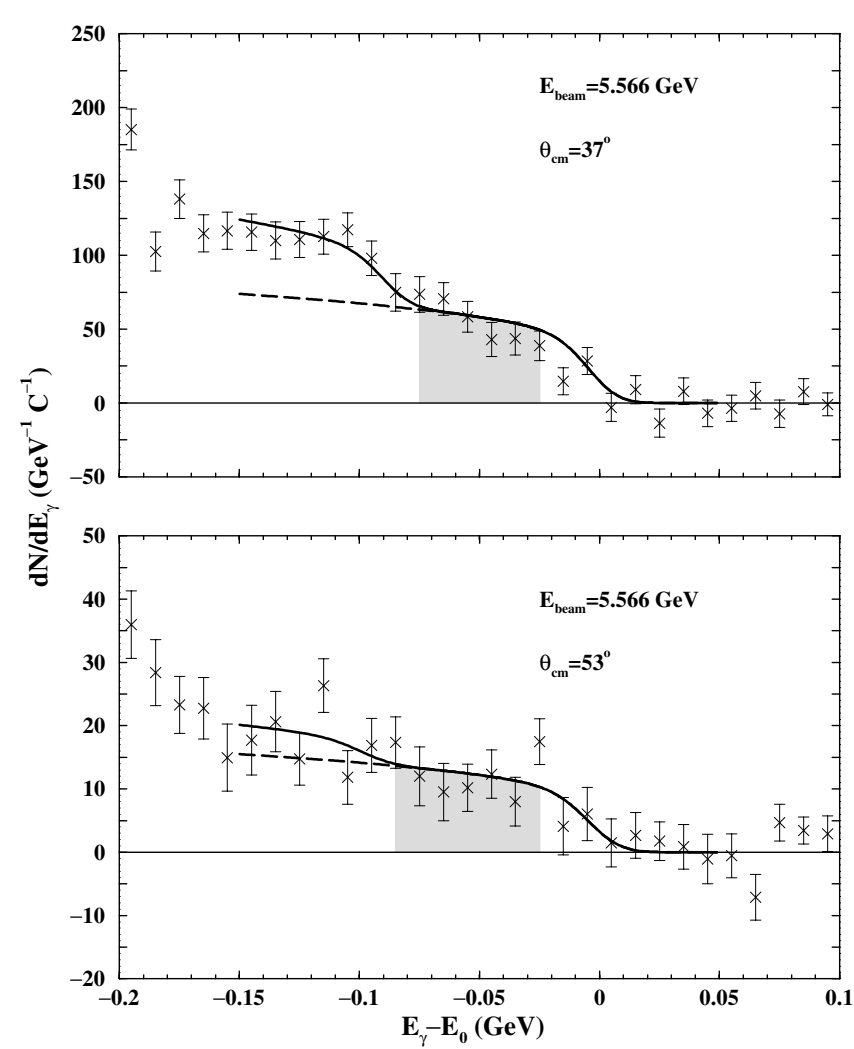

FIG. 1. Photon energy spectra, normalized to collected electron beam charge, for $\theta_{\mathrm{cm}}=37^{\circ}$ (top panel) and $53^{\circ}$ (bottom panel) at $5.5 \mathrm{GeV}$. The grey shaded area denotes the region in $E_{\gamma}$ where the photoproton yield is calculated. The curves are described in the text.

is clearly visible at energies below those highlighted by the shaded region in Fig. 1. There is negligible contamination of the proton yield from the $\pi^{0}$ channel. The grey area just below the end point indicates the region in $E_{\gamma}$ from which the photoproton yield is determined.

In order to properly normalize the photoproton yield and obtain a laboratory cross section, the total number of real bremsstrahlung photons was calculated using the thicktarget bremsstrahlung calculation of Matthews and Owens. This calculation of the photon flux is accurate to 3\% [19]. Because the radiator always remained in the beam line, real bremsstrahlung from the upstream aluminum target window and the cryogenic deuterium must be determined. These radiators were $\$ 2 \%$ of a radiation length, so the photon flux was calculated using the thin target approximation. The electron beam energy distribution was altered after passing through the radiator; consequently, photon yields from components after the radiator were corrected for radiative losses.

Events caused by electrodisintegration of the deuteron were not directly subtracted [4]. These events were compensated for by calculating the virtual photon flux, caused by electrons interacting with the deuterons in the target, and contributed approximately one-third to the total photon yield. An analytical spectrum which includes the recoil of light nuclei, derived by Wright and Tiator [20], was used to determine the total number of virtual photons. The virtual and real photon numbers were added to get the total photon number needed to normalize the photoproton yield properly. Systematic error caused by the use of the Wright and Tiator virtual photon spectrum was determined to be about $5 \%$ by comparing this method with the traditional method of subtracting the electroproduced events [16,21].

Corrections applied to the data included $2 \%$ for tracking efficiency and $\sim 10 \%$ for proton absorption in the detector. The solid angle was determined by a Monte Carlo model of the HMS. The validity of the Monte Carlo was checked by comparing electron-proton $(e-p)$ elastic scattering data with world values. The effect of the extended target was investigated by analyzing $e-p$ elastic scattering data from both 4 and $12.5 \mathrm{~cm}$ liquid hydrogen targets, and agreed to within $2.5 \%$.

The overall systematic error is $\$ 10 \%$. Uncertainty in beam current, beam energy, target length, and photon energy reconstruction in $s^{11} \frac{d \sigma}{d t}$ is $\$ 3.5 \%$. Particle identification and background subtraction contributed approximately $4 \%$ additional uncertainty. All other uncertainties including solid angle determination added $\$ 8.9 \%$, dominated by the $5 \%$ systematic error from the virtual photon technique.

The final results are shown in Fig. 2, plotted as $s^{11} \frac{d \sigma}{d t}$. Three lower energy data points were taken during E96-003.

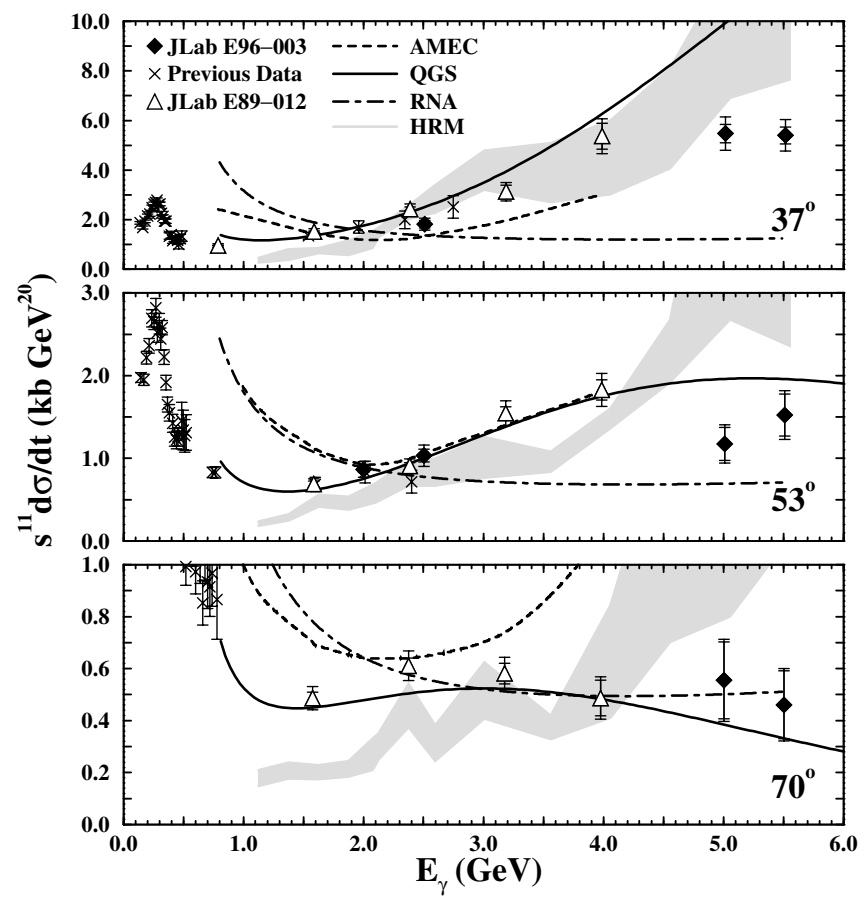

FIG. 2. $\quad s^{11} \frac{d \sigma}{d t}$ vs $E_{\gamma}$ for $d(\gamma, p) n$. The present data are shown as solid diamonds. Errors for JLab data are statistical and total errors. All others are statistical only. E89-012 data are shown as open triangles. All other data are shown as crosses and are as presented in Refs. [2,3,22]. The solid line is the QGS calculation [10]. The long-short dashed line is the RNA calculation [11]. The short dashed line is the AMEC [15]. The grey area is the HRM [9]. 
These results, $\theta_{\mathrm{cm}}=53^{\circ}$ at $E_{\gamma}=2.0 \mathrm{GeV}, \theta_{\mathrm{cm}}=37^{\circ}$ and $53^{\circ}$ at $E_{\gamma}=2.5 \mathrm{GeV}$, overlapped both the JLab E89-012 and SLAC NE17 data and agree quite well with the previous cross section measurements near these energies and angles. Also shown are results from Jefferson Lab experiment E89-012 [4] and data from previous experiments $[2,3,22]$.

QGS gives reasonable agreement with the present results only for $53^{\circ}$ and $70^{\circ}$ above $E_{\gamma}=4 \mathrm{GeV}$ (see Fig. 2). Above $E_{\gamma}=4 \mathrm{GeV}$, RNA is consistent with the energy dependence of these results (see Fig. 2). HRM, however, gives an inadequate description of both the energy and angular dependence of the data in the same region. Both AMEC and traditional meson exchange calculations (see Ref. [4]) need to be extended above $E_{\gamma}=4 \mathrm{GeV}$ in order to evaluate their descriptions.

A normalized $s^{-n}$ fit to all $\frac{d \sigma}{d t}$ data points for $E_{\gamma}>$ $1 \mathrm{GeV}$ at $\theta_{\mathrm{cm}}=70^{\circ}$ resulted in $n=10.8 \pm 0.1$ consistent with constituent counting rules. At $\theta_{\mathrm{cm}}=53^{\circ} \mathrm{a}$ similar fit for data above $E_{\gamma}=3 \mathrm{GeV}$ produced an $n=$ $11.0 \pm 0.3$ suggesting the onset of scaling above $E_{\gamma}=$ $3 \mathrm{GeV}$. In the same energy range at $\theta_{\mathrm{cm}}=37^{\circ}$, a value $n=9.9 \pm 0.2$ indicates that scaling does not set in at $E_{\gamma} \lesssim 3 \mathrm{GeV}$; however, above $E_{\gamma}=4 \mathrm{GeV}$ the fit gives $n=11.0 \pm 0.4$ consistent with constituent counting.

Brodsky and Hiller, in the RNA model, propose transverse momentum, $P_{T}$, as the kinematic quantity responsible for the onset of scaling behavior [11]. $P_{T}$ for $d(\gamma, p) n$ is found from the following expression: $P_{T}^{2}=$ $\frac{1}{2} M_{d} E_{\gamma} \sin ^{2}\left(\theta_{\mathrm{cm}}\right)$. The data indicate apparent scaling thresholds at $E_{\gamma}=3 \mathrm{GeV}$ for $\theta_{\mathrm{cm}}=53^{\circ}$ and $E_{\gamma}=4 \mathrm{GeV}$ for $\theta_{\mathrm{cm}}=37^{\circ}$. From these values an average $P_{T}=$ $1.3 \mathrm{GeV} / c$ with a standard deviation of $0.1 \mathrm{GeV} / c$ is obtained. This could indicate that small transverse size $(\sim 0.15 \mathrm{fm})$ in the deuteron determines the onset of scaling.

These data are the first measurements of the $d(\gamma, p) n$ differential cross section up to $E_{\gamma}=5.5 \mathrm{GeV}$. Results at $\theta_{\mathrm{cm}}=70^{\circ}$ remain consistent with $s^{11 \frac{d \sigma}{d t}}$ scaling previously observed in JLab E89-012. At $\theta_{\mathrm{cm}}=53^{\circ}$ there appears to be a scaling threshold for $E_{\gamma} \geqslant 3 \mathrm{GeV}$. At $\theta_{\mathrm{cm}}=37^{\circ}$, the most forward angle measured, the results are consistent with the $s^{11} \frac{d \sigma}{d t}$ behavior for $E_{\gamma}>4 \mathrm{GeV}$. Improved high energy measurements should be performed to confirm whether the suggested scaling thresholds exist. Further theoretical work in this nonperturbative regime will be necessary to understand the transition region between the meson exchange picture and the QCD description of high energy nuclear reactions.

The authors extend their thanks to the staff of the Accelerator Division at Jefferson Lab for the delivery of high quality 5.0 and $5.5 \mathrm{GeV}$ beams. Thanks is extended also to the Hall $\mathrm{C}$ technical staff for the maintenance and repair of the HMS and its detectors and the members of the Target Group for the installation of the cryotargets. This work is supported by the United States Department of Energy and the National Science Foundation.

* Present address: Physics Division, Argonne National Laboratory, Argonne, IL 60439.

†Present address: St. Mary's University, Halifax, NS, Canada.

[1] R. J. Holt, Phys. Rev. C 41, 2400 (1990).

[2] J. E. Belz et al., Phys. Rev. Lett. 74, 646 (1995).

[3] S. J. Freedman et al., Phys. Rev. C 48, 1864 (1993).

[4] C. Bochna et al., Phys. Rev. Lett. 81, 4576 (1998).

[5] K. Wijesooria et al., Phys. Rev. Lett. 86, 2975 (2001).

[6] S. J. Brodsky and B. T. Chertok, Phys. Rev. D 14, 3003 (1976); Phys. Rev. Lett. 37, 269 (1976); S. J. Brodsky, C-R. Ji, and G. P. Lepage, Phys. Rev. Lett. 51, 83 (1983); L. C. Alexa et al., Phys. Rev. Lett. 82, 1374 (1999); D. Abbott et al., Phys. Rev. Lett. 82, 1879 (1999).

[7] S. J. Brodsky and G. R. Farrar, Phys. Rev. Lett. 31, 1153 (1973); V. Matveev et al., Nuovo Cimento Lett. 7, 719 (1973).

[8] G. P. Lepage and S. J. Brodsky, Phys. Rev. D 22, 2157 (1980).

[9] L. L. Frankfurt et al., Phys. Rev. Lett. 84, 3045 (2000).

[10] V. Y. Grishina et al., Eur. Phys. J. A 10, 355 (2001); L. A. Kondratyuk et al., Phys. Rev. C 48, 2491 (1993); E. De Sanctis et al., Phys. Rev. C 42, 1764 (1990).

[11] S. J. Brodsky and J. R. Hiller, Phys. Rev. C 28, 475 (1983).

[12] A. V. Radyushkin (private communication).

[13] T.S.-H. Lee, Argonne National Laboratory Report No. PHY-5253-TH-88; Proceedings of the International Conference on Medium and High Energy Nuclear Physics, Taipei, Taiwan, 1988 (World Scientific, Singapore, 1988), p. 563.

[14] J. M. Laget, Nucl. Phys. A312, 265 (1978).

[15] A. E. L. Dieperink and S. I. Nagorny, Phys. Lett. B 456, 9 (1999); S. I. Nagorny et al., Sov. J. Nucl. Phys. 55, 189 (1992).

[16] B.P. Terburg, Ph.D. thesis, University of Illinois, 1999 (unpublished).

[17] J. R. Arrington, Ph.D. thesis, California Institute of Technology, 1998 (unpublished).

[18] R. J. Holt, University of Illinois, CEBAF Proposal No. PR-96-003, 1996.

[19] J. L. Matthews and R. O. Owens, Nucl. Instrum. Methods 111, 157 (1973).

[20] L. E. Wright and L. Tiator, Phys. Rev. C 26, 2349 (1982).

[21] C. W. Bochna, Ph.D. thesis, University of Illinois, 1999 (unpublished).

[22] P. Dougan et al., Z. Phys. A 276, 55 (1976); R. Crawford et al., Nucl. Phys. A603, 303 (1996). 